\title{
Estudio termogravimétrico y modelado de la difusión de oxígeno en $\mathrm{YBa}_{2} \mathrm{Cu}_{3} \mathrm{O}_{7-\delta}$
}

\author{
$M^{a}$ D. VÁZQUEZ-NAVARRO, A. KURŠUMOVIÇ ${ }^{1}$, J.E. EVETTS ${ }^{2}$ \\ ${ }^{1}$ Dept. of Materials, Imperial College, Prince Consort Rd., London SW7 2BP, U.K. \\ ${ }^{2}$ Dept. of materials, University of Cambridge, Pembroke St., Cambridge CB2 3QZ, U.K
}

\begin{abstract}
Este trabajo presenta un estudio de la difusión de oxígeno en $\mathrm{YBa}_{2} \mathrm{Cu}_{3} \mathrm{O}_{7-\delta}(\mathrm{YBCO})$ utilizando análisis termogravimétrico diferencial. Una comparación de los resultados experimentales con una de las soluciones de la segunda ley de Fick ha permitido calcular el valor del coeficiente de difusión de oxígeno en este material. Este trabajo se ha completado con el desarrollo de un modelo de elementos finitos que describe el proceso de difusión, ya sea en condiciones isotérmicas o a temperatura variable. El modelo calcula no sólo los cambios de peso, sino también la evolución de la distribución de oxígeno a medida que éste va penetrando en la muestra. El desarrollo de este programa permite estudiar teóricamente los efectos de diferentes procedimientos de oxigenación, pudiendo comprobar la eficacia de diferentes tiempos de oxigenación y la distribución final de oxígeno en la muestra. Esta información se podría utilizar posteriormente para diseñar tratamientos térmicos que den lugar a muestras con un contenido y distribución de oxígeno decidido de antemano.
\end{abstract}

Palabras clave: $\mathrm{YBa}_{2} \mathrm{Cu}_{3} \mathrm{O}_{7-\delta^{\prime}}$ no-estequiometría, coeficiente de difusión, oxigenación isoterma y no-isoterma.

Thermogravimetric study and modelling of oxygen diffusion in $\mathrm{YBa}_{2} \mathrm{Cu}_{3} \mathrm{O}_{7-\delta}$

This paper presents a study of oxygen diffusion in $\mathrm{YBa}_{2} \mathrm{Cu}_{3} \mathrm{O}_{7-\delta}(\mathrm{YBCO})$ using differential thermogravimetric analysis. Comparing the results of the experimental data to those from a solution of Fick's second law, it has been possible to calculate an expression for the chemical diffusion coefficient of oxygen in YBCO. This work is complemented with the results from a theoretical diffusion model developed using finite element modelling to study the diffusion process. This model calculates not only the fractional weight change of the sample during oxygenation, but it also determines the change in distribution of oxygen within the sample during the oxygenation process. The model presented provides a convenient basis for preliminary analyses of any proposed oxygenation procedure, to check oxygenation times and the final distribution of oxygen within the sample. This gives the opportunity to design different heat treatments that give rise to samples with the required oxygen concentration and distribution.

Keywords: $\mathrm{YBa}_{2} \mathrm{Cu}_{3} \mathrm{O}_{7-\delta^{\prime}}$ non-stoichiometry, diffusion coefficient, isothermal and non-isothermal oxygenation.

\section{INTRODUCCIÓN}

Las propiedades de los superconductores de alta temperatura dependen en gran parte de su estequiometría. Para obtener una muestra con un alto contenido de oxígeno se suele mantener entre los 300 y $500{ }^{\circ} \mathrm{C}$ en aire o en oxígeno puro. La duración de esta oxigenación está determinada por el coeficiente de difusión, $\tilde{D}$, la microestructura y la historia térmica de la muestra. Desde el descubrimiento de estos materiales se han realizado muchos estudios de la difusión de oxígeno en $\mathrm{YBa}_{2} \mathrm{Cu}_{3} \mathrm{O}_{7-\delta}(\mathrm{YBCO})$, pero no se ha obtenido un resultado definitivo para este proceso. Se ha observado que los datos publicados hasta la fecha varían hasta en siete órdenes de magnitud (ver referencias (1-6) entre otras).

La razón de los diferentes resultados depende de varios factores: en primer lugar, dependiendo de la técnica utilizada se pueden medir varios tipos de coeficientes de difusión: difusión de isótopos, autodifusión y difusión química. En segundo lugar, la microestructura de la muestra juega un papel importante, puesto que no es lo mismo medir la difusión en un monocristal que en un material policristalino. En el último caso hay que tener en cuenta, por ejemplo, que la distancia de difusión está establecida por el tamaño de grano de los cristales que forman la muestra (en el caso de una muestra porosa) y que, dependiendo de la temperatura, la difusión en las fronteras de grano puede en ocasiones contribuir al proceso general de difusión en la muestra. Las referencias (7) y (8) presentan un resumen de los resultados presentes en la literatura considerando las distintas técnicas utilizadas y los tipos de coeficiente de difusión medidos con cada una.

Este trabajo presenta los resultados del estudio de la difusión química de oxígeno, D̃, en muestras de YBCO en polvo utilizando análisis termogravimétrico. Los resultados se han comparado con los obtenidos teóricamente utilizando el modelo desarrollado para estudiar este proceso de difusión. Los resultados teóricos reproducen con bastante fidelidad los obtenidos experimentalmente, independientemente de si la muestra se ha oxigenado a temperatura constante o bajo condiciones de temperatura variable. 


\section{PROCEDIMIENTO EXPERIMENTAL}

El polvo de YBCO utilizado tiene un diámetro que oscila entre las 2 y $10 \mu \mathrm{m}$, y su contenido de oxígeno inicial es $\sim 7$ (fuente: Seattle Speciality Ceramics). Los experimentos se han realizado utilizando un analizador termogravimétrico PerkinElmer System $7\left(\leq 1000^{\circ} \mathrm{C}\right)$. Las muestras $(\sim 30 \mathrm{mg})$ se desoxigenaron previamente en $\mathrm{Ar}$ a $750{ }^{\circ} \mathrm{C}$ y se oxigenaron después en una atmósfera de oxígeno puro con el siguiente tratamiento: calentamiento rápido a $90{ }^{\circ} \mathrm{C} / \mathrm{min}$ hasta $300,350,400$ ó 450 ${ }^{\circ} \mathrm{C}$. Cada muestra se mantuvo a la temperatura de oxigenación hasta que se observó una estabilización del peso de la misma. Posteriormente las muestras se enfriaron hasta temperatura ambiente a $90^{\circ} \mathrm{C} / \mathrm{min}$.

\section{RESULTADOS Y DISCUSIÓN}

El estudio de los datos experimentales se ha realizado comparándolos con la solución de la segunda ley de Fick aplicada al caso de difusión en una hoja plana. Las condiciones de contorno utilizadas han sido las siguientes: la composición inicial de la muestra es constante y en cuanto ésta alcanza la temperatura de oxigenación, la composición de oxígeno en la superficie es constante e igual a la estequiometría de equilibrio de oxígeno característica de la temperatura elegida. La solución a la segunda ley de Fick bajo estas condiciones es:

$$
\frac{M_{t}}{M_{\infty}}=1-\frac{8}{\pi^{2}} \sum_{n=0}^{\infty} \frac{1}{(2 n+1)^{2}} \exp \left(-D \frac{(2 n+1)^{2} \pi^{2} t}{l^{2}}\right)
$$

donde $M_{t}$ es el peso de la muestra en el tiempo $t, M_{\infty}$ el peso de la muestra para $t=\infty, \tilde{D}$ es el coeficiente de difusión química y $l$ el grosor de la muestra (10). La elección de esta solución bidimensional está justificada, puesto que la difusión de oxígeno en YBCO ocurre principalmente en el plano basal $a b$ de la celda unidad (la difusión a lo largo del eje $c$ es $\approx 10^{6}$ veces más lenta (1), (7)) y por lo tanto el problema se puede simplificar a un caso en dos dimensiones.

La figura 1 muestra una comparación entre el resultado experimental de una oxigenación a $300{ }^{\circ} \mathrm{C}$ y el resultado teórico calculado utilizando la solución de la ley de Fick. Se puede ver que la correspondencia entre ambas curvas es bastante buena. La distancia de difusión $l=5.2 \mathrm{~mm}$ ha sido tomada de acuerdo con la distribución de tamaño de partículas en el polvo y corresponde al diámetro de la partícula más abundante.

Calculando el valor del coeficiente de difusión química a varias temperaturas (utilizando el método de comparación entre los resultados experimentales y los teóricos), ha sido posible obtener la siguiente expresión de Arrhenius que describe el proceso: $\tilde{D}=0.033 \exp [-1.24(\mathrm{eV}) / \mathrm{kT}]$ (ver figura 2).

\section{MODELO DEL PROCESO DE DIFUSIÓN}

Para completar este estudio se ha desarrollado un programa que reproduce la evolución del proceso de difusión (11). Tomando la primera ley de Fick como punto de partida: $F=\tilde{D} \frac{\partial c}{\partial x}$, donde $\mathrm{F}$ es el flujo de especie que difunde por unidad de área, $\tilde{D}$ el coeficiente de difusión, $c$ es la concentración de la especie y $x$ la distancia recorrida perpendicular a la sección considerada, se puede calcular el cambio en el perfil de concentración de oxígeno por unidad de tiempo dentro de

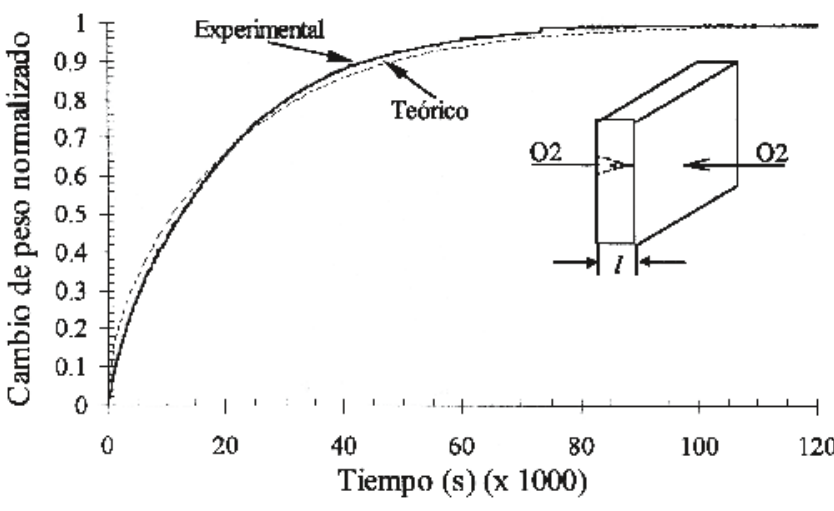

Figura 1. Comparación entre el resultado experimental y el teórico calculado a partir de la ley de Fick para una oxigenación a $300{ }^{\circ} \mathrm{C} . \quad \tilde{D}$ $=3 \times 10-13 \mathrm{~cm}^{2} / \mathrm{s}$. Dimensión de la hoja plana para el cáclulo de la solución teórica: $l=5.2 \mathrm{~mm}$. Este grosor corresponde con el tamaño más abundante en la distribución de tamaño de partículas del polvo utilizado.

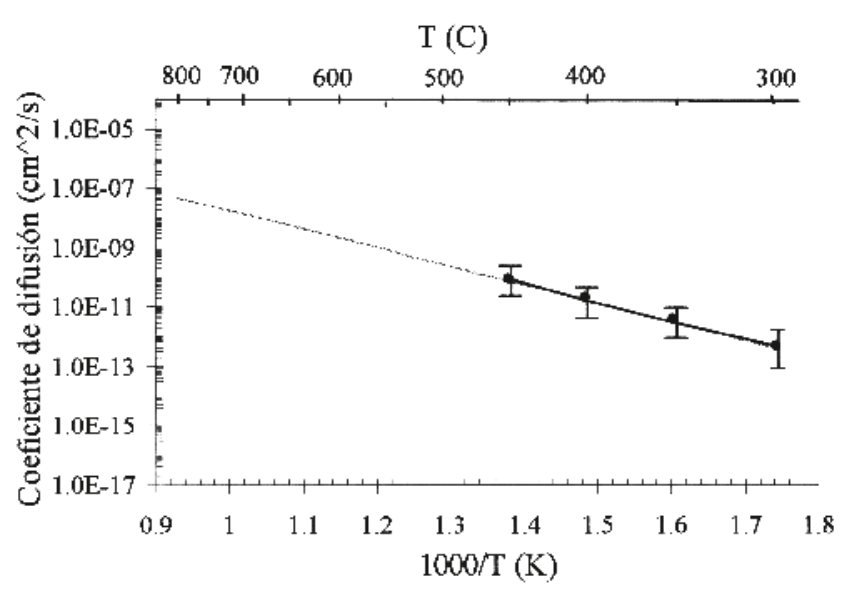

Figura 2. Valores del coeficiente de difusión química, $\tilde{D}$, calculados de acuerdo con la solución a la segunda ley de Fick correspondiente a la difusión en una hoja. El gráfico incluye los márgenes de error en los cálculos realizados.

cada elemento del sistema, puesto que la cantidad neta de la especie que difunde $\mathrm{q}_{\mathrm{net}}$ (de oxígeno en este caso) acumulada en cada elemento del sistema es igual a la diferencia entre la cantidad de oxígeno que ha entrado en el elemento, $\mathrm{q}_{\mathrm{r}}$ menos la cantidad que pasa de ese elemento al siguiente, $\mathrm{q}_{\mathrm{s}}$ (ver la figura 3 a)) (9). Esta cantidad se puede expresar como:

$$
q_{n e t}=q_{r}-q_{s}=-\frac{\widetilde{D} \tau}{d x} A\left(2 C_{1}-C_{0}-C_{2}\right)
$$

donde $\tau$ es el intervalo de tiempo considerado, $\mathrm{C} 1$ es la concentraciones en el centro y $\mathrm{C} 0$ y C2 son las concentraciones de oxígeno a ambos lados de los elementos considerados, como se muestra en la figura 3 a).

En este programa se supone que las partículas de YBCO son esféricas y éstas se dividen en discos paralelos de un grosor $d h$. Cada uno de estos discos está dividido en círculos concén- 


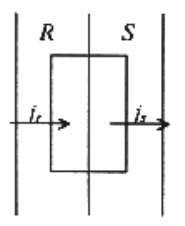

a)
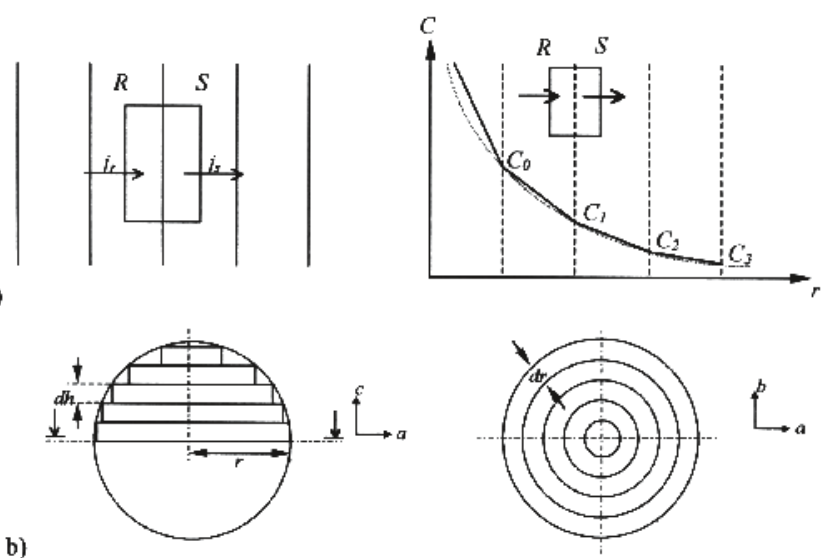

Figura 3. a) Esquema de la primera ley de Fick; b) División de la partícula esférica en discos de grosor $d h$ (gráfico de la izquierda) y división de los discos en cilindros concéntricos separados una distancia $d r$.

tricos separados una distancia $d r$, y la difusión ocurre en el plano de los discos de grosor $d h$ hacia el centro de los mismos (Figura $3 \mathrm{~b}$ )).

\subsection{Oxigenación en condiciones isotérmicas}

La figura 4 a) muestra una comparación entre la curva experimental de difusión de oxígeno en una muestra de YBCO en polvo a $350{ }^{\circ} \mathrm{C}$ y el cambio de peso normalizado que se ha obtenido utilizando el modelo desarrollado. Se puede observar que ambos resultados son muy similares.

Este modelo no sólo reproduce los cambios de peso durante la oxigenación sino que también calcula el cambio en el perfil de difusión dentro de la muestra (ver figura 4 b)). Ésto proporciona información sobre la distribución de oxígeno en la muestra después del ciclo de oxignación utilizado y podría permitir saber cómo cambiaría esta distribución al modificar el tratamiento térmico de la oxigenación.

\subsection{Oxigenación en condiciones no-isotérmicas}

La oxigenación en condiciones no-isotérmicas presenta más problemas porque la muestra se encuentra en todo momento en una situación de no-equilibrio. Esto se debe al cambio de la estequiometría de oxígeno de YBCO con la temperatura (6). La figura 5 muestra el perfil característico de una oxigenación experimental de $\mathrm{YBCO}$ en polvo a $50{ }^{\circ} \mathrm{C} / \mathrm{min}$ hasta $700{ }^{\circ} \mathrm{C}$. La dependencia del coeficiente de difusión de oxígeno con la temperatura junto con la estequiometría variable de YBCO permiten explicar la forma de esta curva: a temperaturas bajas la difusión de oxígeno es muy lenta y por lo tanto no se observa ninguna ganancia de oxígeno a la velocidad a la que se está calentando la muestra. Una vez que el valor de $\tilde{D}$ ha aumentado lo suficiente $\left(\mathrm{T} \sim 300{ }^{\circ} \mathrm{C}\right)$, se ve que la muestra va ganando peso hasta que llega un momento en que lo empieza a perder. En este punto el valor del coeficiente de difusión de oxígeno en el YBCO es tan rápido que el peso de la muestra corresponde en todo momento al contenido de equilibrio de oxígeno del material, que disminuye con el aumento de temperatura.

A pesar de estar en una situación de no-equilibrio durante la mayor parte de la oxigenación, la reproducción teórica de los datos experimentales es bastante buena, como se puede

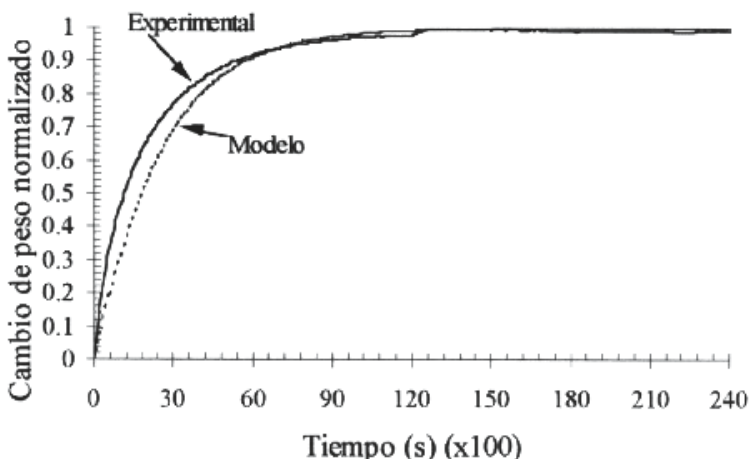

a)

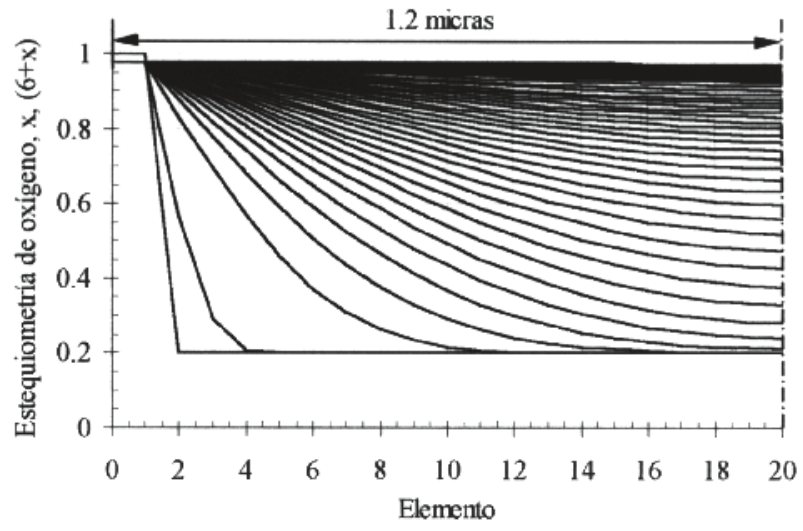

b)

Figura 4. a) Oxigenación de polvo de $\mathrm{YBCO}$ a $350{ }^{\circ} \mathrm{C}$. Comparación entre los datos obtenidos experimentalmente (línea contínua) y el resultado del modelo de elementos finitos desarrollado (línea discontínua). $\tilde{D}=1.9 \times 10^{-12} \mathrm{~cm}^{2} / \mathrm{s} ; \mathrm{b}$ ) Evolución de los perfiles de concentración de oxígeno en la muestra para la misma oxigenación a $350^{\circ} \mathrm{C}$. Tiempo entre perfiles de concentración: $5.5 \mathrm{~min}$. Este esquema muestra el perfil de concentración sólo en una mitad de la muestra, puesto que la distribución es simétrica respecto al centro (elemento 20).

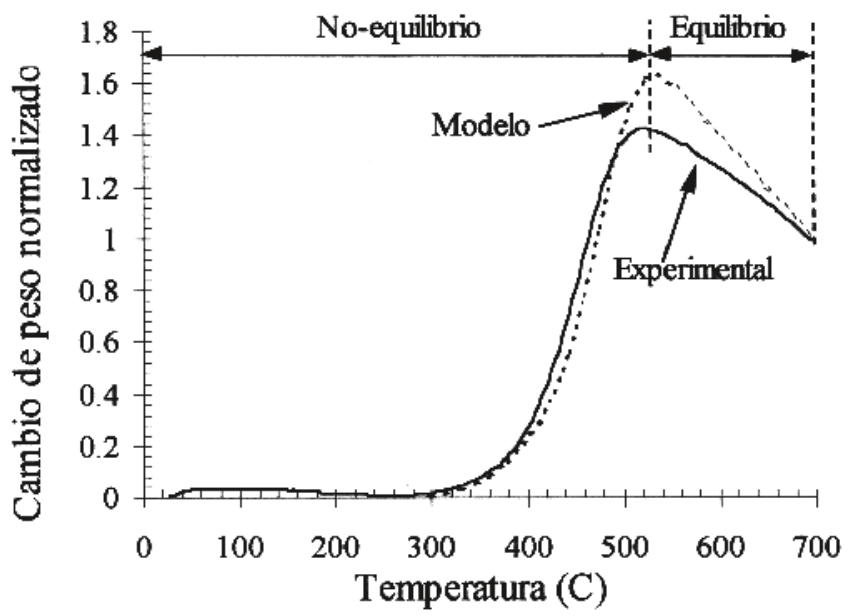

Figura 5. Comparación entre el resultado experimental (línea continua) y el teórico (línea discontínua) de una oxigenación de polvo de YBCO a $50{ }^{\circ} \mathrm{C} / \mathrm{min}$ hasta $700{ }^{\circ} \mathrm{C}$. 


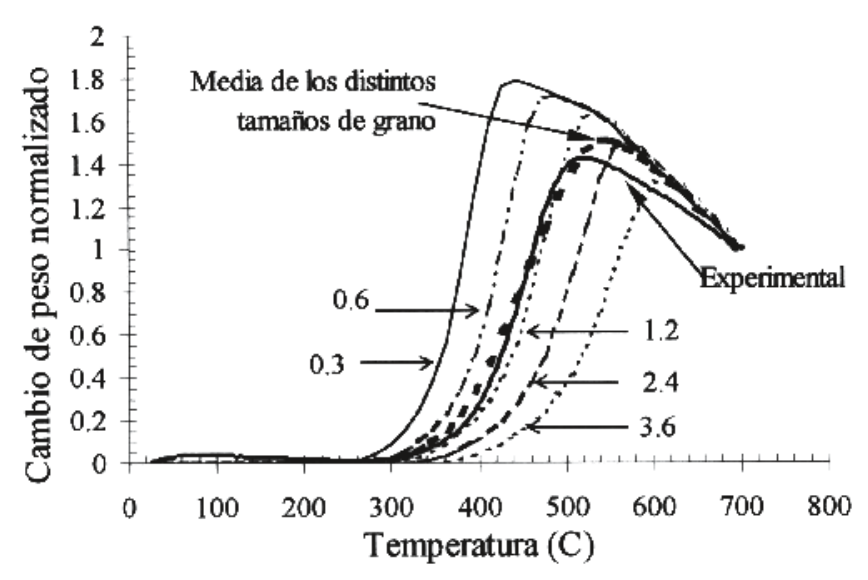

Figura 6. Curvas de oxigenación obtenidas para diferentes tamaños de partícula (en micras) y su media (línea gruesa discontínua) comparadas con los datos experimentales (línea gruesa contínua). Tratamiento: $50{ }^{\circ} \mathrm{C} / \mathrm{min}$ de $25{ }^{\circ} \mathrm{C}$ a $700{ }^{\circ} \mathrm{C}$.

ver en la misma figura 5. Se puede observar que el máximo en la curva es de mayor magnitud en el caso del cálculo teórico; ésto se puede atribuir a la distribución del tamaño de partícula en el polvo (8) y (9). La figura 6 muestra cómo haciendo una media entre los resultados de diferentes tamaños de partícula se obtiene una curva próxima a los resultados experimentales. En este modelo no se ha tenido en cuenta el cambio de los mecanismos de difusión con la temperatura.

\section{CONCLUSIONES}

Este trabajo ha mostrado brevemente los resultados obtenidos en el cálculo del coeficiente de difusión de oxígeno en $\mathrm{YBa}_{2} \mathrm{Cu}_{3} \mathrm{O}_{7-\delta}$ utilizando la técnica de análisis termogravimétrico. El cálculo de la expresión de Arrhenius que rige el proceso de difusión en este material, junto con un análisis de elementos finitos del proceso de difusión, ha permitido desarrollar un modelo teórico que se ha utilizado para reproducir oxigenaciones a temperatura constante y variable. El programa desarrollado para estudiar teóricamente el proceso de difusión es muy versátil, puesto que:
1) La reproducción teórica de oxigenaciones experimentales a temperatura constante y variable es muy buena.

2) Se podría utilizar para predecir el cambio de peso después de un determinado tratamiento térmico, pudiendo saber la distribución de oxígeno en la muestra después del tratamiento. De este modo se podrían optimizar y diseñar de antemano las oxigenaciones para obtener muestras de YBCO completamente oxigenadas en eel mínimo espacio de tiempo.

\section{AGRADECIMIENTOS}

Este proyecto se ha realizado con una beca del Consejo de Investigación EPSRC de Gran Bretaña con una beca CASE (Cooperación en Ciencia e Ingeniería) otorgada en colaboración con la empresa EA Technology (GB). Los autores agradecen a Dr. J. Ruiz las sugerencias para el diseño del modelo de difusión.

\section{BIBLIOGRAFÍA}

1. S.J. Rothman, J.L. Routbort, U. Welp, J.E. Baker, "Anisotropy of oxygen tracer diffusion in single-crystal $\mathrm{YBa}_{2} \mathrm{Cu}_{3} \mathrm{O}_{7-\delta}$ ", Phys. Rev. B, 44, 2326-2331 (1991)

2. K.N.Tu, N.C. Yeh, S.I. Park, C.C. Tsuei, "Diffusion of oxygen in $\mathrm{YBa}_{2} \mathrm{Cu}_{3} \mathrm{O}_{7-\delta}$ superconducting ceramic oxides", Phys. Rev. B, 39, 304-314, (1989).

3. J.L. Tallon, M.P. Staines, "Oxygen self-diffusion coefficient in the superconductor $\mathrm{YBa}_{2} \mathrm{Cu}_{3} \mathrm{O}_{7-\delta}$ from internal friction measurements", J. Appl. Phys 68, 3999-4001, (1990).

4. B.A. G_owacki, R.J. Highmore, K.F. Peters, A.L. Greer, J.E. Evetts, "A calorimetric study of oxygen intercalation and desorption in bulk superconducting $\mathrm{YBa}_{2} \mathrm{Cu}_{3} \mathrm{O}_{7-\mathrm{x}}$ ", Supercond. Sci. and Techn., 1, 7-11, (1988).

5. S. Tsukui, T. Yamamoto, M. Adachi, Y. Shono, K. Kawabata, N. Fukoka, S. Nakanishi, A. Yanase, Y. Yoshioka, "Direct observation of ${ }^{18} \mathrm{O}$ tracer diffusion in a $\mathrm{YBa}_{2} \mathrm{Cu}_{3} \mathrm{O}_{\mathrm{y}}$ single crystal by secondary ion mass spectrometry", Jpn. J. Appl. Phys., 30, L973-L976, (1991).

6. K. Kishio, K. Suzuki, T. Hasegawa, T. Yamamoto, K. Kitazawa, "Study on chemical diffusion of oxygen in $\mathrm{Ba}_{2} \mathrm{YCu}_{3} \mathrm{O}_{7-\delta}$ ", 82, 192-202, (1989).

7. J.L. Routbort, S.J. Rothman, "Oxygen diffusion in cuprate superconductors", J. Appl. Phys., 76, 5615-5628, (1994).

8. M.D. Vázquez-Navarro, Tesis doctoral: "A thermogravimetric study of oxygen diffusion in $\mathrm{YBa}_{2} \mathrm{Cu}_{3} \mathrm{O}_{7-\delta}$ ", Universidad de Cambridge (1998).

9. M.D. Vázquez-Navarro, A. Kuršumoviç, C. Chen , J.E. Evetts, "Analysis and modelling of oxygen diffusion in $\mathrm{YBa}_{2} \mathrm{Cu}_{3} \mathrm{O}_{7-\mathrm{x}}$ under non-isothermal conditions", Inst. Phys. Conf. Ser. No. 158, 1037-1040, (1997).

10. J. Crank, "The mathematics of diffusion", $2^{\text {nd }}$ Edition, Oxford Science Publications (1975).

11. M.D. Vázquez-Navarro, A. Kur_umoviç, J.E. Evetts, "Study and modelling of oxygen diffusion in $\mathrm{YBa}_{2} \mathrm{Cu}_{3} \mathrm{O}_{7-\delta}$ under isothermal conditions", Supercond., Sci., Technol., 12, 1117-1122, (1999). 